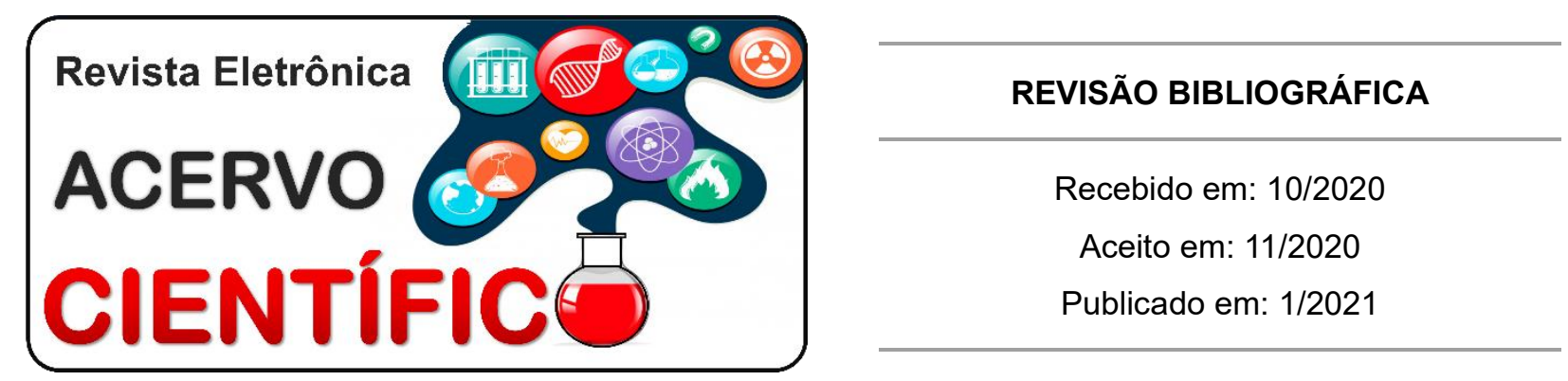

\title{
Transtorno do Espectro Autista na percepção de acadêmicos universitários: uma revisão de literatura
}

\author{
Autistic Spectrum Disorder in the perception of university academics: a literature review \\ El Trastorno del Espectro Autista em la percepción de los académicos universitários: una \\ revisión de la literatura
}

Layandra Vittória de Assis ${ }^{1 *}$, Gustavo Fonseca Genelhu Soares², Adrielle Almeida Quixabeira ${ }^{3}$, Bruna Carolina Rangel Fortes ${ }^{4}$, Carolina Nunes Caro Varela ${ }^{5}$, Guilherme Pesolitto Hubinger ${ }^{5}$, Hiann Werner Braun Oliveira de Melo², Leonardo Antonio Vieira Lisboa², Mariana Duque Coelho Barros Baía², Matheus Filipe de Souza Lima².

Resumo: Esse artigo tem o objetivo de discutir como o Transtorno do Espectro Autista (TEA) influencia no desenvolvimento psicossocial dos estudantes universitários dentro do espectro, analisando suas experiências acadêmicas e sociais. O TEA é considerado um transtorno do neurodesenvolvimento que pode compreender prejuízo na interação social e comunicação, além de apresentar comportamentos repetitivos e interesses restritos. Ao longo dos anos, a inserção de indivíduos com TEA nas universidades apresentou um aumento, por efeito de iniciativas legais e do apoio ao estudante, além da maior conscientização, reconhecimento e número de diagnósticos. $O$ trabalho trata-se de uma revisão bibliográfica, na qual foi analisada a experiência universitária de estudantes com TEA, e a importância de ações que visem o suporte e a permanência dessa população na universidade. As especificidades dos indivíduos dentro do espectro tornam evidente a necessidade de suporte e estratégias que propiciem inclusão no ambiente social acadêmico, com o intuito de melhorar seu envolvimento social e qualidade de vida.

Palavras-chave: Transtorno do Espectro Autista, Deficiências da aprendizagem, Estudantes.

\begin{abstract}
This article aims to discuss how Autistic Spectrum Disorder (ASD) influences the psychosocial development of university students within the spectrum, analyzing their academic and social experiences. ASD is considered a neurodevelopmental disorder that can include impairment in social interection and comunnication, in addition to presenting repetitive behaviors and restricted interests. Over the years, the inclusion of individuals with ASD in universities has increased, dua to legal initiatives and student support, in addition to greater awareness, recognition and the number of diagnoses. The work is a bibliographic review, where the university experience of students with ASD was anlyzed and the importance of actions aimed at the support and permanence of this population at the university. The specificities of the individuals within the spectrum make evident the need for support and strategies that provide inclusion in the academic social environment, in order to improve their social involvement and quality of life.
\end{abstract}

Keywords: Autistic Spectrum Disorder, Learning disabilities, Students.

\footnotetext{
1 Instituto Metropolitano de Ensino Superior de Vale do Aço (IMES UNIVAÇO), Ipatinga - MG.

*E-mail: layandraassis@yahoo.com.br

2 Centro Universitário de Caratinga (UNEC), Caratinga - MG.

3 Universidade de Rio Verde (UniRV), Rio verde - GO.

${ }^{4}$ Centro Universitário Presidente Antônio Carlos (UNIPAC), Juiz de Fora - MG.

${ }^{5}$ Universidade Nove de Julho (UNINOVE), São Paulo - SP.
} 
Resumen: Este artículo tiene como objetivo discutir cómo el trastorno del espectro autista (TEA) influye en el desarrollo psicosocial de los estudiantes universitarios dentro del espectro, analizando sus experiencias académicas y sociales. EI TEA se considera un trastorno del neurodesarrollo que puede comprender perjuicio en la interacción social y la comunicación, además de presentar comportamientos repetitivos e intereses disminuidos. A lo largo de los años, la inserción de individuos con TEA en las universidades presentó un incremento, por efecto de las iniciativas legales y apoyo al estudiante, además de una mayor conciencia, reconocimiento y número de diagnósticos. Este trabajo es una revisión bibliográfica, donde la experiencia universitaria de los estudiantes con TEA, y la importancia de acciones que mejoran el soporte y la permanencia de esta población en la universidad. Las especificidades de los individuos dentro del espectro hacen evidente la necesidad de apoyos y estrategias que promuevan la inclusión en el ámbito social académico, con la para mejorar su implicación social y calidad de vida.

Palabras clave: Trastorno del Espectro Autista, Discapacidades para el aprendizaje, Estudiantes.

\section{INTRODUÇÃO}

O Transtorno do Espectro do Autismo (TEA) é uma desordem neurológica caracterizada por déficit na interação social, alteração na comunicação e padrões limitados ou estereotipados de comportamentos e interesses. Esse transtorno pode ocorrer com ou sem deficiência intelectual, permitindo que indivíduos classificados no espectro um, com capacidade cognitiva funcional, sejam capazes de experimentar uma série de desafios sociais que se relacionam com os critérios de diagnóstico do TEA (EAVES LC e HO HH, 2008).

A observação das experiências acadêmicas e sociais em estudantes universitários com Transtorno do Espectro Autista é importante para melhor avaliar uma forma de fornecer apoio e desenvolver oportunidades para aumentar o envolvimento social e a qualidade de vida desses estudantes (VAN HEIJST BF e GEURTS HM, 2015). Haja vista que a prevalência de jovens adultos no espectro do autismo a ingressar na vida universitária na última década resultou em uma maior atenção sobre como melhor atender às necessidades de ensino dessa população crescente (HILL A, et al., 2015).

De acordo com um estudo recente, $48 \%$ dos estudantes universitários autistas estavam satisfeitos com sua carga de trabalho acadêmica e se consideravam bem-sucedidos academicamente (GELBAR NW, et al., 2015). No entanto, alguns alunos do espectro relataram suas experiências, as quais indicavam que a velocidade de processamento de informações, gerenciamento de tempo, trabalhos em grupo, apresentações, motivação para estudar, seguir palestras e fazer perguntas podem ser desafios significativos (VAN HEES V, et al., 2015).

Pesquisas apresentadas na Jornada Internacional de Inclusão Educacional apontaram que alunos autistas relatam que a estrutura em ambientes acadêmicos, instruções concretas e tarefas menores os ajudaram a lidar com alguns dos desafios na graduação (CAI RY e RICHDALE A, 2016). Foram observados também outros pontos positivos relacionados aos estudantes que se encontram dentro do espectro como memória proeficiente, foco em detalhes, pensamentos criativos, interesses intensos, desejo de adquirir conhecimento e adesão clara à regra. Essas habilidades podem impactar positivamente as experiências acadêmicas e os resultados para alunos autistas (VAN HEES V, et al., 2015).

O objetivo do presente estudo consiste em analisar a produção científica sobre a associação das experiências acadêmicas e sociais em estudantes universitários com Transtorno do Espectro Autista, buscando a relação do transtorno no desenvolvimento psicossocial dos pacientes. Além disso, visa comparar estes resultados a pesquisas realizadas no mundo ocidental.

\section{REVISÃO BIBLIOGRÁFICA}

Segundo a Associação Americana de Psicologia, o Transtorno do Espectro Autista (TEA) é uma patologia que altera o neurodesenvolvimento, causando prejuízos constantes nas relações sociais, nas interações e na comunicação, além de demonstrar um comportamento restrito e repetitivo. Embora as causas do Transtorno do Espectro Autista ainda sejam desconhecidas pela comunidade científica e não haja consenso para explicar essa condição, existem estudos que associam sua origem à interação gene-ambiente ou, ainda, causada por 
fatores genéticos (ALHARBI KA, et al., 2019). De acordo com as formas encontradas, notou-se a heterogeneidade no desenvolvimento de indivíduos com TEA, ou seja, enquanto a grande parte das crianças que receberam o diagnóstico de TEA na infância continuam a demonstrar dificuldades sociais evidentes na fase adulta, um outro grupo não foi capaz de preencher os critérios diagnósticos após a infância (OLIVATI AG, et al., 2019).

De acordo com o Manual Diagnóstico e Estatístico de Transtornos Mentais (DSM-5), publicado pela Associação Americana de Psiquiatria, no que se refere à escala do nível de gravidade da doença, observamse três níveis importantes (SILVA SC, et al., 2019). O Nível um, é a forma mais branda da doença, havendo necessidade de apoio contínuo nas comunicações sociais, no padrão de comportamento repetitivo e restrito, gerando uma dificuldade elevada na realização do diagnóstico (MARTINS ADF e MONTEIRO MIB, 2017). Já no Nível dois, existe necessidade de apoio substancial, ou seja, nota-se um significativo prejuízo social e uma dificuldade maior em estabelecer diálogos iniciais, apresenta comportamento inflexível e pode se estressar com facilidade. O Nível três, necessita de apoio muito substancial, sendo o comprometimento mais severo, onde a criança apresenta graves prejuízos na comunicação, elevado grau de estresse e irritabilidade se exposto a uma mudança de foco ou atividade (AMERICAN PSYCHIATRIC ASSOCIATION, 2013).

Estima-se que uma a cada 59 crianças americanas com 8 anos são diagnosticadas com o transtorno. Já na maior faixa etária, aproximadamente 50.000 adolescentes com autismo entram na idade adulta a cada ano nos Estados Unidos e 1\% de toda a população adulta inglesa é afetada pelo Transtorno do Espectro Autista. Entre as pessoas no espectro autista, $69 \%$ não possuem deficiência intelectual, e $46 \%$ têm capacidade intelectual média ou superior. Jovens com TEA apresentam independência subdesenvolvida, dificuldade nas habilidades interpessoais, prejuízo na capacidade de controlar o estresse e emoções intensas, padrões de pensamentos obsessivos, dificuldade de manter conversas em ambientes sociais, interpretar pistas não verbais e a perspectiva de outras pessoas, o que pode agravar as dificuldades em fases de transição (HODGES H, et al., 2020; ANDERSON HA, et al., 2017; WHITE SW, et al., 2019; BRUGHA TS, et al., 2011; PINDER-AMAKER S, 2014). Segue abaixo a Tabela 1 formulada pelos autores do respectivo artigo para detalhar as características no espectro autista.

Tabela 1 - Características específicas do autismo e respectiva porcentagem de responsividade mais comuns em jovens e crianças autistas.

\begin{tabular}{|l|c|}
\hline Características específicas do autismo & $\begin{array}{c}\text { Porcentagem } \\
\text { correta da } \\
\text { responsividade }\end{array}$ \\
\hline $\begin{array}{l}\text { 1. Comprometimento de comportamentos não verbais, como contato visual, } \\
\text { impressões faciais, gestos, postura e linguagem corporal }\end{array}$ & 86,9 \\
\hline 2. Comportamentos estereotípicos e repetitivos & 66,9 \\
\hline 3. Olhar fixamente sem concentração para um objeto & 61,1 \\
\hline 4. Aparência estática e estereótipo de surdez & 56,3 \\
\hline 5. Grande desempenho em atividades de rotina & 54,3 \\
\hline 6. Desatenção ao mundo exterior & 53,1 \\
\hline 7. Hábito alimentar anormal & 48,6 \\
\hline 8. Não compartilhar interesses ou certar atividades espontaneamente & 43,4 \\
\hline 9. Não ter facilidade para sorrir socialmente & 33,7 \\
\hline
\end{tabular}

Fonte: Assis LV, et al., 2020. Dados do extraídos de Lüleci NE, et al., 2016.

Ao redor do mundo, um número significativo de alunos nas escolas são afetados pelo autismo. As escolas públicas têm um nível superior de conhecimento. O nível educacional, grau de ensino, experiência, gênero demonstraram não serem fatores que implicam no conhecimento dos professores (BAMBARA LM, et al., 2018).

As características de aprendizagem para este grupo são exclusivas e bem diferentes do que é oferecido para a grande maioria dos alunos, exigindo que professores tenham habilidades especializadas e preparem com excelência o conteúdo a ser administrado, para que possa ser prestado um serviço de auxílio em âmbito social e de ensino. A gestão do autismo infantil e outras formas generalizadas de transtornos do 
desenvolvimento requer serviços profissionais também como psiquiatras, pediatras e psicólogos, entre outros (IGWE MN, et al., 2010). Vale ressaltar que jovens com TEA de alto funcionamento, apesar de intelectualmente capazes de buscar educação superior, possuem maior probabilidade de encontrar desafios psicossociais na mudança do ensino médio para a faculdade (ALHARBI KA, et al., 2019; PINDER-AMAKER S, 2014).

À medida que atingem a adolescência, o uso da linguagem falada em situações sociais, para os indivíduos com Transtorno do Espectro do Autismo, assume uma grande importância. No Ensino Médio e na vida adulta, as interações cotidianas com os colegas giram em torno de rodas de conversa. No entanto, devido às dificuldades pelo pragmatismo da doença, adolescentes com TEA muitas vezes resistem pela demanda crescente por conversas sociais, aumentando seu risco de isolamento, rejeição de colegas e ansiedade social, que também pode ser resultante do reconhecimento de sua deficiência na habilidade comunicativa e de envolvimento social (BAMBARA LM, et al., 2018; VAN SCHALKWYK GI e VOLKMAR FR, 2017).

A transição para a idade adulta corresponde ao estágio de desenvolvimento em que há um provável aumento de tolerância, aceitação e abertura para a diversidade. Dessa forma, durante a fase acadêmica os alunos estão mais dispostos a se aproximarem e terem relacionamentos com indivíduos com TEA. Embora estejam menos propensos a sustentarem uma vida social em comum, ainda conseguem manter vínculos estudantis, o que facilita sua inserção no ambiente universitário, e possibilita um maior sucesso social e acadêmico (GARDINER E e IAROCCI G, 2014).

A inserção de pessoas com Transtorno do Espectro Autista (TEA) em universidades aumentou no decorrer dos anos devido ao apoio prestado aos estudantes e a iniciativas legais que auxiliam o ingresso do públicoalvo da educação especial, além do aumento da conscientização, reconhecimento e diagnósticos de TEA. Apesar do número do grupo de autistas no ensino superior ser pequeno em relação aos estudantes neurotípicos, é perceptível o aumento de matrículas de pessoas com deficiência. O perfil de estudantes universitários foi modificado a partir da promulgação da lei 12.711/2012, que dispõe do recurso de reserva de vaga para pessoas com deficiência, assim como para autodeclarados pretos, pardos e indígenas em universidades federais. Propiciando assim, o aumento do ingresso de estudantes com TEA em instituições públicas federais, bem como a redução de protótipos de padrões sociais excludentes (SILVA SC, et al., 2019).

O período universitário compreende o momento com maior incidência de doenças psiquiátricas importantes, como transtorno depressivo maior, mesmo em indivíduos neurotípicos. Acadêmicos com TEA possuem um risco duas vezes maior de desenvolver condições psiquiátricas. Estudos sugeriram que estudantes no espectro podem possuir vulnerabilidade elevada aos estressores, o que se associa com sua condição (PINDER-AMAKER S, 2014).

De acordo com Chandrasekhar T (2019), pesquisas com estudantes universitários no espectro relataram uma variedade de condições psiquiátricas, como altas taxas de ansiedade, depressão, solidão, poucas amizades íntimas e TDAH.

Apesar do ensino público e privado possuírem programas governamentais que facilitam o ingresso ao ensino superior, o número de estudantes com TEA é majoritário na rede privada, e a maioria opta por realizar o curso de forma presencial. O estudo presencial apresenta ônus e bônus aos estudantes autistas, os quais apresentam dificuldade de comunicação e sociabilidade, no ambiente acadêmico estarão inseridos em um espaço que necessita de integração com colegas e professores, o que pode resultar em relações positivas ou acarretar em sofrimento. $O$ ensino a distância, entretanto, permite um ensino flexível e facilita a aprendizagem, pois o estudante com TEA que possui sensibilidade sensorial estará apto a estudar em um ambiente em que se sinta confortável (SILVA SC, et al., 2019).

No contexto da inclusão em ambiente acadêmico, o papel do profissional da educação é imprescindível. O professor deve compreender as singularidades do estudante no espectro para adequar sua metodologia para que o aprendizado aconteça. Pode utilizar recursos como a comunicação clara sem a utilização de figuras de linguagem, a flexibilidade no tempo de entrega de trabalhos, e a supressão de ações que causem um mal estar sensorial no estudante. Além disso, o educador pode tornar-se um facilitador para que a socialização do aluno com TEA aconteça entre os demais (ROCHA BR, et al., 2018). 
O estudante universitário com TEA carece de estratégias que propiciem o aprendizado, como utilização de materiais pedagógicos adequados, adequações na linguagem, organização e atividades pedagógicas, assim como a necessidade de uma tutoria que permita o desenvolvimento de habilidades sociais. Todavia, os investimentos em ações que combatem atitudes preconceituosas, materiais adequados e qualificação de professores e funcionários que promovam o desenvolvimento do aluno são escassos (SILVA SC, et al., 2019).

Atualmente poucos estudos e pesquisas se dedicam às necessidades de estudantes com TEA, as pesquisas são essenciais para que haja o desenvolvimento de programas que possam atender as necessidades dos portadores. Sendo assim, os estudos existentes indicam que a maioria dos estudantes recebem suporte educacional, mas não social. Para o ingresso no ensino superior é preciso que o estudante receba acompanhamento e suporte, no entanto a transição do ensino secundário não é planejada previamente e o aluno ao adentrar a universidade não revela seu diagnóstico a instituição. Apesar do transtorno estar associado com a deficiência intelectual, cerca de $50 \%$ dos estudantes possuem um QI na faixa de normalidade. Ademais, apresentam déficit social, comportamentos repetitivos, sensibilidade sensorial e dificuldades cognitivas como ansiedade e depressão (CAI RY e RICHDALE AL, 2015).

De forma concomitante ao TEA, a maioria dos estudantes possuem ansiedade, depressão e transtorno obsessivo compulsivo. Com isso, para reduzir os sintomas da ansiedade os alunos procuram mecanismos para reduzi-la como praticar a leitura. Muitos deles não são organizados, fato que pode influenciar de forma negativa o desempenho acadêmico. Um dos motivos da desorganização é a facilidade em se distrair e a dificuldade em obter foco ao realizar multitarefas. Além disso, os estudantes com TEA não conseguem resolver as avaliações e tarefas de sala em tempo adequado pois possuem dificuldade em absorver novas informações, dessa forma demandam de maior tempo para realizar as atividades (CAI RY e RICHDALE AL, 2015).

Devido aos déficits recorrentes, poucos adultos com TEA possuem um bom emprego ou resultados educacionais, e não são capazes de atingir seu potencial acadêmico devido ao isolamento social, estresse excessivo e dependência de apoio familiar. Por possuírem deficiência em comunicação social, os estudantes não participam de discussões em grupo, caso contrário pode passar por grande estresse. Altos níveis de estresse e ansiedade desencadeiam um comportamento agressivo, e para que o quadro seja revertido os alunos necessitam de um suporte acadêmico apropriado, como ajustes e flexibilidade no estilo de ensino (CAI RY e RICHDALE AL, 2015).

O Decreto 7.611/2011 instituiu a estruturação de núcleos de acessibilidade nas Instituições Federais de Educação Superior, dessa forma torna-se evidente a necessidade da valorização do processo de inclusão social para que além da possibilidade de ingresso no ensino superior, os estudantes com deficiência tenham condições de permanência e sucesso acadêmico. O sistema educacional deve condizer às necessidades específicas de cada estudante em relação à aprendizagem para que o aluno possa se beneficiar da educação e adquirir bons resultados (SILVA SC, et al., 2019).

Muitos estudantes são diagnosticados na metade do curso ou apenas ao terminá-lo. Há alunos que possuem um diagnóstico e não recebem nenhum apoio por falta de instruções ou por não divulgar o diagnóstico. Dessa forma, enfrentam problemas como falta de amigos, dificuldade em realizar tarefas em grupo, isolamento, dificuldade para planejamento, de cumprimento de horários, e dificuldades em realizar provas devido a sensação de não possuir tempo suficiente para responder as perguntas e à sensibilidade sensorial. Além das barreiras enfrentadas na universidade, os alunos precisam lidar com a mudança de residência, aprender a morar sozinho, frequentar novos ambientes e ser capaz de tomar decisões. Esses aspectos dependem de capacidade de auto regulação e gestão, mas os indivíduos com TEA são deficientes neste aspecto (ANUNCIBAY RDF e GÓMEZ JLC, 2017).

Para que o estudante com déficit receba o suporte necessário é preciso que haja o diagnóstico correto do transtorno e que o aluno ou responsável divulgue essa informação à instituição de ensino superior. Muitos alunos optam por não divulgar que possuem TEA por medo de discriminação, bullying ou pela crença de que não possuem mais deficiência. O suporte quando aplicado de forma correta propicia melhores resultados acadêmicos, apoio em tempos de crise, educação sobre TEA para docentes, apoio individualizado ao aluno, 
e aconselhamento para tratamento de ansiedade e depressão. Os estudos apontam que os serviços de apoio à deficiência nas universidades são insuficientes para atender todas as necessidades dos alunos com TEA (CAI RY e RICHDALE AL, 2015).

Novas formas de apoio institucional têm sido sugeridas, como serviços de aconselhamento, suporte de comunicação social, acomodação em quarto individual, mentores de pares e acomodações acadêmicas. A modelagem em vídeo para ensino de habilidades em comunicação tem demonstrado eficácia significativa, mesmo quando utilizada de forma singular. A tutoria por pares é uma estratégia que visa ajudar os alunos com TEA em seus resultados acadêmicos e sociais. Consiste em um programa de mentoria de aprendizagem autorregulada que possibilita uma média de notas e taxa de retenção mais alta. Neste método um aluno que não possui deficiência se torna voluntário em interagir com um aluno com TEA para ajudá-lo. Ambos os alunos são beneficiados pois os indivíduos com TEA reduzem a ansiedade, melhoram as habilidades sociais e hábitos de estudo, já os seus pares aprendem novas formas de aprendizagem e desenvolvem empatia, paciência e sensibilidade (GARDINER E e IAROCCI G, 2014; CAI RY e RICHDALE AL, 2015; MCLEOD JD, et al., 2019; WHITE SW, et al., 2019).

\section{CONSIDERAÇÕES FINAIS}

Observou-se um aumento no ingresso de alunos diagnosticados com transtorno do espectro autista em universidades, principalmente na rede privada e em aulas na modalidade presencial. Devido ao grande estresse no meio acadêmico, foi constatado o aparecimento de condições psiquiátricas como depressão, ansiedade e agressividade nesse público. Além disso, a solidão, dificuldades em realizar tarefas em grupo, poucas amizades e discriminação foram queixas constantes desses alunos. Medidas têm sido implementadas para que o aluno com TEA atinja seu potencial, mas ainda é necessária uma melhor compreensão da realidade vivenciada por alunos universitários com TEA para que ferramentas mais efetivas sejam utilizadas, de modo que se haja um melhor aproveitamento acadêmico por essas pessoas.

\section{REFERÊNCIAS}

1. ALHARBI KA, et al. School's Teachers Knowledge About Autism in Al-Badayacity, Al-Qassim Region, Kingdom of Saudi Arabia. Mater Sociomed, 2019; 31(1): 4-9.

2. AMERICAN PSYCHIATRIC ASSOCIATION, 2013. In: Autism spectrum disorder.

3. ANDERSON AH, et al. Perspectives of University Students with Autism Spectrum Disorder. J Autism Dev Disord, 2018; 48(3): 651-665.

4. ANUNCIBAY RDF, GÓMEZ JLC. Inclusión de alumnado con trastorno del espectro del autismo en la universidad: análisis y respuestas desde una dimensión internacional. Revista INFAD de Psicología. International Journal of Developmental and Educational Psychology, 2017; 4(1): 13-22.

5. BAMBARA LM, et al. Improving the assertive conversational skills of adolescents with autism spectrum disorder in a natural context. Research in Autism Spectrum Disorders, 2018; 48: 1-16.

6. BRUGHA TS, et al. Epidemiology of Autism Spectrum Disorders in Adults in the Community in England. Arch Gen Psychiatry, 2011; 68(5): 459-465.

7. CAI RY, RICHDALE A. Educational Experiences and Needs of Higher Education Students with Autism Spectrum Disorder. Journal of autism and developmental disorders, 2016; 46: 31-41.

8. CHANDRASEKHAR T. Supporting the needs of college students with autism spectrum disorder. J Am Coll Health, 2019; $1: 1-4$.

9. EAVES LC, HO HH. Young adult outcome of autism spectrum disorders. J Autism Dev Disord, 2008; 38(4):739-47.

10. GARDINER E, IAROCCI G. Students with Autism Spectrum Disorder in the University Context: Peer Acceptance Predicts Intention to Volunteer. J Autism Dev Disord, 2014; 44: 1008-1017.

11. GELBAR NW, et al. A comprehensive survey of current and former college students with autism spectrum disorders. Yale J Biol Med, 2015; 88(1):45-68.

12. HILL A, et al. Epidemiology of Autism Spectrum Disorders. Translational Approaches to Autism Spectrum Disorder, 2015; 1: 13-38.

13. HODGES $\mathrm{H}$, et al. Autism spectrum disorder: definition, epidemiology, causes, and clinical evaluation. Transl Pediatr, 2020; 9(1): 55-65. 
14. IGWE MN, et al. Factors influencing knowledge about childhood autism among final year undergraduate Medical, Nursing and Psychology students of University of Nigeria, Enugu State, Nigeria. Ital J Pediatr, 2010; 1: 36-44.

15. MARTINS ADF, MONTEIRO MIB. Alunos autistas: análise das possibilidades de interação social no contexto pedagógico. Psicologia Escolar e Educacional, 2017; 21(2): 2015-224.

16. MCLEOD JD, et al. The Experiences of College Students on the Autism Spectrum: A Comparison to Their Neurotypical Peers. J Autism Dev Disord, 2019; 49(6): 2320-2336.

17. OLIVATI AG, LEITE LP. Experiências Acadêmicas de Estudantes Universitários com Transtornos do Espectro Autista: uma Análise Interpretativa dos Relatos. Revista Brasileira de Educação Especial, 2019; 25(4), 729-746.

18. PINDER-AMAKER S. Identifying the unmet needs of college students on the autism spectrum. Harv Rev Psychiatry, 2014; 22(2): 125-37.

19. SILVA SC, et al. Perfil acadêmico dos estudantes com Transtorno do Espectro Autista no Ensino Superior matriculados em 2016. Revista Educação Especial, 2019; 32: 1-32.

20. SOUZA BR, et al. Universitários autistas: considerações sobre a inclusão de pessoas com T.E.A nas IES e sobre a figura do docente nesse processo. Revista Educação em Foco, 2018; 9: 140-153.

21. VAN HEES V, et al. Higher Education Experiences of Students with Autism Spectrum Disorder: Challenges, Benefits and Support Needs. J Autism Dev Disord, 2015; 45: 1673-1688.

22. VAN HEIJST BF, GEURTS HM. Quality of life in autism across the lifespan: a meta-analysis. Autism, 2015; 19(2):15867.

23. VAN SCHALKWYK GI, VOLKMAR FR. Autism Spectrum Disorders: Challenges and Opportunities for Transition to Adulthood. Child Adolesc Psychiatr Clin N Am, 2017; 26(2): 329-339.

24. WHITE SW, et al. Improving Transition to Adulthood for Students with Autism: A Randomized Controlled Trial of STEPS. J Clin Child Adolesc Psychol, 2019; 1: 1-15. 